\title{
Associação entre morfologia do ovócito e taxa de fertilização após ICSI
}

\author{
Relationship between oocyte morphology and fertilization rate after ICSI
}

\author{
Alexandros Aggelis ${ }^{1}$, Daniel Faúndes ${ }^{2}$, Alessandra Mattos ${ }^{3}$, Carlos Petta ${ }^{4}$, \\ Paulo Augusto Neves ${ }^{5}$, Aníbal Faúndes ${ }^{6}$
}

\section{RESUM0}

Objetivos: verificar a possibilidade de selecionar ovócitos que resultariam em maior taxa de fertilização. Métodos: estudo retrospectivo que analisou a taxa de fertilização após ICSI de 957 ovócitos em metáfase II segundo três parâmetros da morfologia ovocitária: granulações citoplasmáticas, espaço perivitelino e fragmentação do primeiro corpúsculo polar. Os ovócitos foram obtidos de 115 ciclos realizados em 107 mulheres atendidas no CRHC, entre abril e dezembro de 2004. Para a análise estatística das diferenças na taxa de fertilização entre ovócitos "norm ais" e os que apresentavam cada alteração, utilizou-se o teste de $\chi^{2}$,com nível de confiança de 5 e $10 \%$. Resultados: não se observou diferença significativa na taxa de fertilização segundo as características do corpúsculo polar ou espessura do espaço perivitelino. A taxa de fertilização dos ovócitos com espaço perivitelino apresentando debris foi quase 14 pontos percentuais inferior ao dos ovócitos com espaço "ausente" $(\mathrm{p}=0,055)$ e a dos ovócitos com citoplasma granular foi sete pontos percentuais inferior à obtida pelos ovócitos com citoplasma de aspecto normal $(\mathrm{p}<0,10>0,05)$. Conclusões: os parâmetros da morfologia do ovócito atualmente utilizados não permitem distinguir claramente aqueles que serão fertilizados dos que não serão.

PALAVRAS-CHAVE: Ovócitos; Granulações citoplasmáticas; Espaço perivitelino; Corpúsculo polar; Taxa de fertilização; Fertilização in vitro

\section{ABSTRACT}

Purpose: to verify the possibility of identifying oocytes that would result in a higher fertilization rate. Methods: retrospective analysis of the fertilization rate after ICSI of 957 oocytes in metaphase II according to three morphology parameters: cytoplasm inclusions, thickness of the perivitelline space, and fragmentation of the first polar body. Oocytes were obtained from 115 cycles performed among 107 women attended at the "Centro de Reprodução Humana de Campinas", from April to December of 2004. For the statistical analysis of differences in the fertilization rate between 'norm al' oocytes and those presenting each alteration,the $\chi^{2}$ test was used with confidence levels of 5 and $10 \%$. Results: no significant difference in fertilization rate $w$ as observed regarding characteristics of the polar body or thickness of the perivitelline space. Fertilization rate among oocytes with perivitelline space with debris was 14 percentage points lower than among oocytes with absent space $(\mathrm{p}=0.055)$ and the rate among oocytes with granular cytoplasm was seven percentage points lower than among oocytes with normal cytoplasm $(\mathrm{p}<0.10>0.05)$. Conclusions: the morphological parameters of oocytes currently being evaluated do not allow us to clearly distinguish those that would lead to a higher fertilization rate and could be used in clinical practice.

KEYWORDS: Oocytes; Cytoplasm inclusions; Perivitelline space; Polar body; Fertilization rate; Fertilization in vitro

Centro de Reprodução Humana de Campinas

1 Embriólogo Responsável pelo Laboratório, Centro de Reprodução Humana de Campinas (SP), Brasil.

2 Professor Assistente Doutor, Departamento de Tocoginecologia - Faculdade de Ciências Médicas, UNICAMP. Médico Associado, Centro de Reprodução Humana de Campinas (SP), Brasil.

3 Médica Assistente, Centro de Reprodução Humana de Campinas (SP), Brasil.

4 Professor Livre Docente, Departamento de Tocoginecologia - Faculdade de Ciências Médicas, UNICAMP. Médico Associado, Centro de Reprodução Humana de Campinas (SP), Brasil.

5 Médico Associado, Centro de Reprodução Humana de Campinas (SP), Brasil.

6 Professor Titular Colaborador Voluntário, Departamento de Tocoginecologia - Faculdade de Ciências Médicas, UNICAMP, Médico Associado, Centro de Reprodução Humana de Campinas, Pesquisador Sênior, Centro de Pesquisas em Saúde Reprodutiva de Campinas (SP), Brasil.

Correspondência: Alexandros Aggelis

Centro de Reprodução Humana de Campinas

Rua Dr. Eduardo Lane, 380 - Guanabara - 13075-050 - Campinas - SP - Telefone/Fax: (19) 3243-1317 - e-mail: alex@reproducaohumana.com.br 
Introdução

A tendência mundial dos centros de reprodução assistida é reduzir o número de embriões transferidos ao mínimo, para evitar a gestação múltipla e suas conseqüências para a mãe e para os fetos e recém-nascidos. Além disso, por problemas éticos cada vez mais considerados, tende-se a evitar os embriões excedentes que constituem problema a médio e longo prazo nas clínicas de fertilização assistida. E numa época em que caminhamos para transferência única de embriões, é cada vez mais inapropriado gerar embriões que eventualmente tenham que ser descartados.

O número excessivo de embriões excedentes resulta da fertilização de número maior de ovócitos que o número de embriões desejados. Essa prática se justifica, pois a taxa de fertilização acha-se em torno de 60 a 70 por cento na maioria das clínicas, e ainda, há grande variabilidade individual. $\mathrm{O}$ ideal seria fertilizar apenas o número de ovócitos correspondente aos embriões desejados, o que pode reduzir os custos do laboratório ${ }^{1}$ e aumentar sua eficiência. Mas para isso seria necessário ter certeza razoável de que todos ou quase todos os ovócitos tratados fossem fertilizados. Isto cria a necessidade de escolher quais ovócitos deverão ser injetados, seja por não desejo de congelamento de embriões por parte do casal, seja por existir número grande de ovócitos recuperados.

A introdução da técnica do ICSI (injeção intracitoplasmática de espermatozóides) permitiu obter novas informações sobre as características do ovócito, já que requer a remoção das células do cumulus, o que possibilitou correlacionar a morfologia do ovócito com taxas de fertilização e clivagem e com o desenvolvimento embrionário ${ }^{2}$. A partir dessas informações, vários autores têm proposto que determinadas características dos ovócitos seriam bons indicadores de melhores possibilidades de sucesso na fertilização ou nas taxas de gravidez pós-ICSI ${ }^{1,3-9}$. Entretanto, não há ainda consenso se a morfologia do ovócito permite predizer os resultados da fertilização. Enquanto uns acreditam que certas características da morfologia oocitária se associam a melhores ou piores resultados ${ }^{1,3-7}$, outros sugerem que a morfologia oocitária não afetaria a taxa de fertilização, a qualidade embrionária ou as taxas de gravidez pós-ICSI ${ }^{2,10,11}$.

Para verificar se é possível selecionar ovócitos que resultariam em maior taxa de fertilização, avaliou-se se havia correlação entre essa taxa e três parâmetros da morfologia oocitária: espessura do espaço perivitelino, fragmentação do primeiro corpúsculo polar de ovócitos metáfase II e presença de granulações citoplasmáticas.
Métodos

Foi realizada análise retrospectiva do resultado da fertilização por ICSI de 957 ovócitos em metáfase II obtidos de 115 ciclos realizados em 107 mulheres, entre abril e dezembro de 2004. Pouco mais da metade das mulheres tinham menos de 35 anos (53\%), 27,0\% tinham de 35 a 39 e 20,0\%, 40 anos ou mais. A média de idade das pacientes foi de 33,4 anos (21-45). O fator masculino era responsável exclusivo em $29,0 \%$ dos casos, o fator tubário e endometriose por $12,0 \%$ cada um, ESCA por $9,6 \%$ e outros fatores por $10,4 \%$ dos casos. Em $27,0 \%$ havia mais de um fator presente.

Utilizou-se agonista do hormônio liberador de gonadotrofinas (GnRH-a) para bloqueio hipofisário (Lupron Depot ${ }^{\circledR}, 1 / 2$ ampola de 3,75 mg; Abbott, Rio de Janeiro, RJ, Brasil) no $21^{\circ}$ dia do ciclo anterior. Foi confirmada a supressão ovariana mediante avaliação ultra-sonográfica transvaginal.

A hiperestimulação ovariana foi realizada pela administração de menotropina (hMG: Pergonal ${ }^{\circledR}$, Serono, São Paulo, SP, Brasil) na dose diária de 150 UI associada a hormônio folículoestimulante recombinante (FSH: Gonal- $\mathrm{F}^{\circledR}$, Serono) na dose diária de 150 UI, sendo que a dose de ambas as drogas foi ajustada de acordo com a resposta ovariana. Administrou-se a dose de $250 \mu \mathrm{g}$ de gonadotropina coriônica recombinante (hCG: Ovidrel ${ }^{\circledR}$, Serono) na presença de pelo menos dois folículos com diâmetro médio acima de $18 \mathrm{~mm}$. A captação dos ovócitos foi realizada entre 35 e 36 horas após a administração do hCG. Os ovócitos foram separados do líquido folicular sob estereoscópio e lavados em meio de cultura HTF modificado com HEPES (Irvine Scientific), suplementado com $10 \%$ de soro sintético substitutivo (SSS - Irvine Scientific) antes de serem transferidos em meio de cultura HTF (Irvine Scientific) suplementado com $10 \%$ de SSS e incubados na estufa de $37^{\circ} \mathrm{C} / 5 \% \mathrm{CO}_{2}$ em ar.

A remoção das células do cumulus oophorus foi realizada com hialuronidase (H-4272, Sigma) na concentração de $80 \mathrm{UI} / \mathrm{mL}$ em meio HTF modificado com HEPES suplementado com 10\% SSS, usando micropipetas de vidro. Após a limpeza dos ovócitos, eles permaneceram incubados na estufa em meio de cultura HTF suplementado com 10\% SSS até a realização da ICSI.

As amostras de sêmen foram colhidas logo após a punção folicular, avaliadas na câmara de Makler e processadas em gradiente coloidal Isolate Lower/Isolate Upper ${ }^{\circledR}$ (Irvine Scientific) seguindo o protocolo do fabricante. Um microlitro dos espermatozóides recuperados foi colocado numa gota de 
PVP (Irvine Scientific, 99219). A ICSI foi realizada em microgotas de meio HTF modificado com HEPES suplementado com 10\% SSS num microscópio invertido (Nikon, Eclipse TE300) equipado com micromanipulador (Narishige). Antes da injeção, os ovócitos foram avaliados e fotografados. Foram avaliados os seguintes parâmetros: espessura do espaço perivitelino, integridade do corpúsculo polar e granulação citoplasmática.

O espaço perivitelino foi caracterizado como "ausente" quando o citoplasma estava em contato com a zona pelúcida na maior parte de seu perímetro (Figura 1) e como "aumentado" quando havia espaço entre a zona pelúcida e o citoplasma em mais de $50 \%$ da periferia da zona pelúcida (Figura 1). Apesar de se considerar a ausência de espaço vitelino como "normal", não utilizamos este termo, porque a maior parte dos ovócitos apresentou espaço perivitelino aumentado. O ovócito foi caracterizado como com debris quando se observavam exclusões citoplasmáticas no espaço perivitelino (Figura 1).

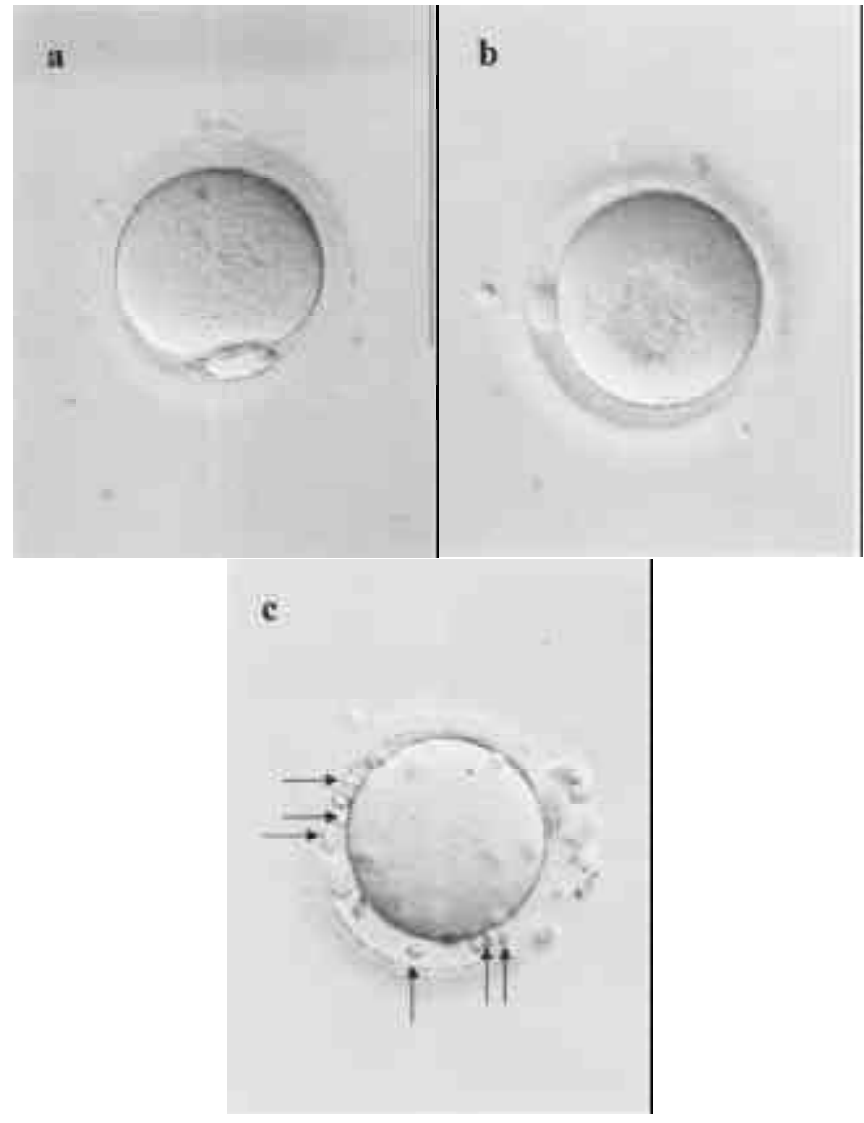

Figura 1 - Espaço perivitelino: a) ausente; b) aumentado; c) aumentado com debris (setas).

O primeiro corpúsculo polar foi caracterizado como "integral" quando se apresentava sem divisões (Figura 2) e como "fragmentado" quando estava dividido em duas ou mais partes (Figura 2).

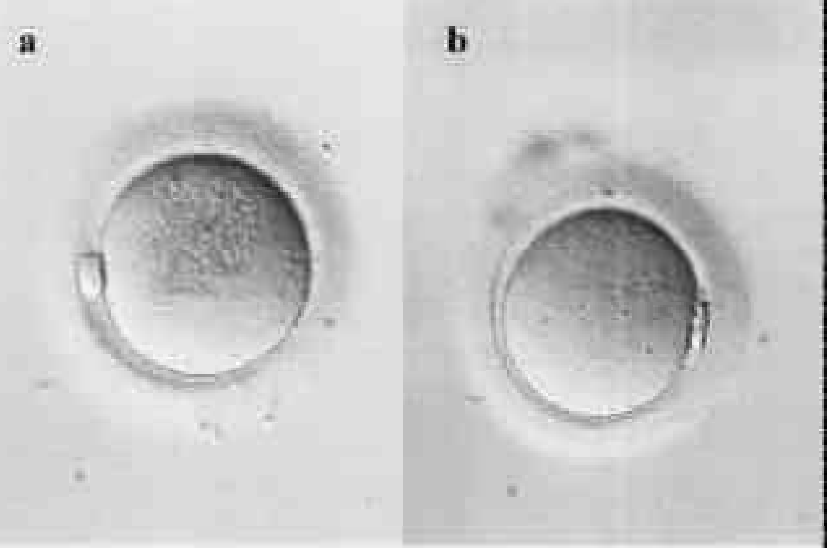

Figura 2 - Morfologia do primeiro corpúsculo polar: a) integral; b) fragmentado.

A qualidade citoplasmática dos ovócitos foi classificada como "granular" se houvesse regiões de citoplasma escuro (Figura 3) e "normal" quando as mesmas não eram observadas (Figura 3).

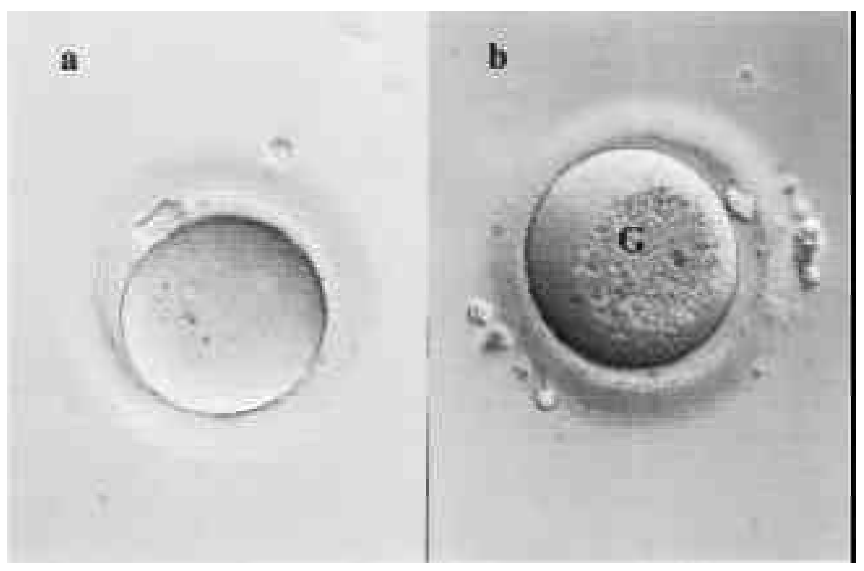

Figura 3 - Oócitos MII com: a) citoplasma normal; b) citoplasma granular (G).

Após ICSI, os óvulos foram transferidos em gotas de 40 microlitros de meio HTF suplementado com 10\% SSS sob óleo mineral (Irvine Scientific, 9305) e foram incubados separadamente até o dia seguinte na estufa. A fertilização foi avaliada 1820 horas após a ICSI. Apenas os zigotos com dois pró-núcleos distintos foram considerados fertilizados.

Foi avaliada a taxa de fertilização obtida entre os ovócitos que apresentavam cada uma das características descritas acima, comparando-a com a taxa observada entre os ovócitos classificados como "normais" quanto a essa alteração. Os ovócitos foram incluídos na análise, tanto no grupo de "normais" como de com uma alteração específica, independentemente de que esses mesmos ovócitos apresentassem ou não, simultaneamente, outras possiveis alterações. Além disso, foi comparada a taxa de fertilização de ovócitos que não apresentavam nenhuma alteração com ovócitos 
que apresentavam uma, duas ou as três das alterações estudadas. Caso um ovócito apresentasse alterações em alguma dessas três características diferentes das descritas acima, esse ovócito era excluído da análise.

Para a análise estatística das diferenças na taxa de fertilização entre ovócitos "normais" e os que apresentavam cada alteração, utilizou-se o teste de $\chi^{2}$, com niveis de confiança de 5 e $10 \%$.

\section{Resultados}

Não se observou diferença significativa na taxa de fertilização segundo as características do espaço perivitelino. Apesar de a taxa de fertilização ter sido ligeiramente inferior nos casos em que o espaço foi classificado como "aumentado" $(67,6 \%)$ em relação com os casos com espaço "ausente" ou normal $(70,4 \%)$, essa diferença não apresentou significância estatística (Tabela 1). Apenas o grupo de ovócitos com espaço apresentando "debris" $(56,9 \%)$ mostrou taxa de fertilização quase 14 pontos percentuais inferior ao dos ovócitos com espaço "ausente", diferença que ficou perto da significância estatística $(p=0,055)$ (Tabela1).

Tabela 1 - Taxas de fertilização segundo as características do espaço perivitelínico.

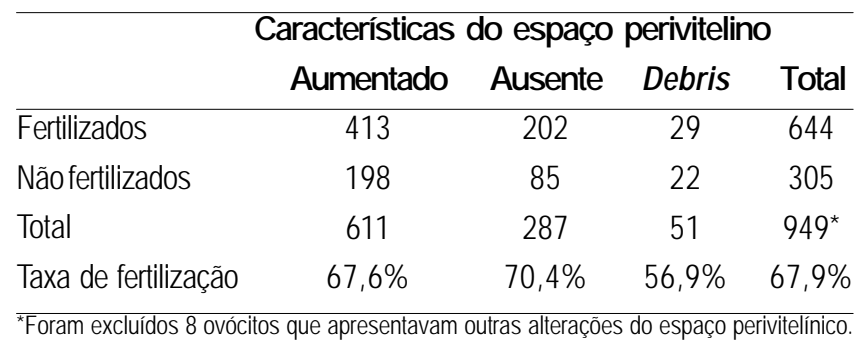

A taxa de fertilização dos ovócitos com corpo polar "fragmentado" $(68,1 \%)$ foi quase exatamente a mesma e até ligeiramente superior à observada para os ovócitos com corpúsculo polar classificado como "integral" $(67,8)$ (Tabela 2$)$.

Tabela 2 - Taxas de fertilização segundo a qualidade do primeiro corpúsculo polar.

\begin{tabular}{lccr}
\hline & \multicolumn{2}{c}{ Corpúsculo polar } & \\
& Fragmentado & Integral & Total \\
\hline Fertilizados & 275 & 353 & 628 \\
Não fertilizados & 129 & 168 & 297 \\
Total & 404 & 521 & $925^{\star}$ \\
Taxa de fertilização & $68,1 \%$ & $67,8 \%$ & $67,9 \%$ \\
\hline
\end{tabular}

A taxa de fertilização dos ovócitos com citoplasma granular $(62,6 \%)$ foi sete pontos percentuais inferior à obtida pelos ovócitos com citoplasma de aspecto normal $(69,7)$. O valor de $\mathrm{p}$ foi $\langle 0,10\rangle$ 0,05 (Tabela 3).

Tabela 3 - Taxa de fertilização segundo a qualidade do citoplasma.

\begin{tabular}{lccc}
\hline & \multicolumn{2}{c}{ Citoplasma } & \\
& Normal & Granular & Total \\
\hline Fertilizados & 529 & 119 & 648 \\
Não fertilizados & 236 & 71 & 307 \\
Total & 765 & 190 & $955^{\star}$ \\
Taxa de fertilização & $69,2 \%$ & $62,6 \%$ & $67,9 \%$
\end{tabular}

${ }^{\star}$ Foram excluídos dois ovócitos que apresentavam outras alterações do citoplasma.

Ao comparar a taxa de fertilização segundo o número de alterações, encontraram-se diferenças significativas entre os ovócitos sem alterações $(69,9 \%)$ e os com uma alteração $(59,9 \%)$, mas não houve diferenças quando comparados com os ovócitos com duas ou mais alterações $(67,6 \%)$ (Tabela 4). A diferença entre os sem alterações e o conjunto dos com uma e duas ou mais alterações também foi estatisticamente significativa, com $\mathrm{p}<0,05$.

Tabela 4 - Taxas de fertilização segundo o número de alterações morfológicas dos ovócitos.

\begin{tabular}{lcccc}
\hline \multicolumn{4}{c}{ Número de alterações } \\
& Zero & Uma & Duas ou mais & Total \\
\hline Fertilizados & 100 & 258 & 252 & 610 \\
Não fertilizados & 44 & 173 & 121 & 338 \\
Total & 144 & 431 & 373 & 948 \\
Taxa de fertilização & $69,4 \%$ & $59,9 \%$ & $67,6 \%$ & $64,3 \%$
\end{tabular}

Zero vs 1; $p=0,041$; zero vs 2 ou +: $p=0,680$. Mantel e Haenszel para o conjunto; $p=0,088$.

\section{Discussão}

Os resultados desta análise sugerem que é possível que algumas características dos ovócitos possam ter correlação com as taxas de fertilização. Entretanto, a diferença na taxa de fertilização obtida de ovócitos com características de normalidade comparados com aqueles com alterações poderá chegar a ser estatisticamente significativa, mas não permite identificar ovócitos que tenham possibilidades tão elevadas de fertilização que resultem de utilidade para sua utilização na prática clínica. Por outra parte, ovócitos morfologicamente deficientes resultam em taxas de fertilização relativamente elevadas, dentro dos padrões descritos na maior parte das publicações, 
pelo que resulta difícil recomendar sua não utilização para ICSI.

A ausência de correlação significativa entre as características do espaço perivitelino e taxa de fertilização em nossos casos está de acordo com a literatura. Publicações dos últimos 10 anos encontraram mínima diferença, e estatisticamente não significativa, entre ovócitos com espaço "espesso" e "normal" (Tabela 5). A maior diferença, ainda que não significativa, foi encontrada por De Sutter et al. ${ }^{11}$, mas com um número relativamente pequeno de casos. Recalculando os dados de $\mathrm{Xia}^{4}$, baseado nos números da Tabela 2 desse trabalho, encontramos resultados muito similares aos nossos, com taxas de fertilização de $73,3 \%$ para ovócitos com espaço normal e 67,4\% para os com espaço espesso, com diferenças também não significativas. O maior número de casos na literatura foi apresentado por Balaban et al. ${ }^{10}$, que encontraram mínima diferença na taxa de fertilização segundo características do espaço perivitelino $(72,9$ e $71,8 \%)$. Somando os casos dos três autores citados acima, mais os nossos casos, incluímos perto de 3.000 ovócitos com espaço "normal" e mais de 1.700 com espaço "espesso", obtendo uma diferença ainda pequena $(72,6$ e $69,3 \%$, respectivamente), mas que atinge significação estatística, com $\mathrm{p}<0,02$. Esta significação estatística, no entanto, não se traduz em significação clínica, já que uma diferença na taxa de fertilização de 69 para $72 \%$ não permite utilizar essa característica do ovócito para desprezar os primeiros e praticar ICSI apenas nos segundos. Além disso, somar os casos dos quatro estudos não é totalmente aceitável, visto que o critério para definir o espaço perivitelino como "normal" ou "espesso" é subjetivo, não está explícito em nenhum dos trabalhos revisados e pode ser diferente entre eles. Mostra-se apenas uma fotografia como exemplo, obviamente selecionada entre os casos em que essa característica oferecia menos dúvidas de classificação.

Tabela 5 - Taxa de fertilização segundo as características do espaço perivitelino.

\begin{tabular}{lccccc}
\hline Autor & \multicolumn{3}{c}{$\begin{array}{c}\text { Espaço perivetilino } \\
\text { Normal }\end{array}$} & p \\
& $\mathbf{n}$ & \% Fértil & $\mathbf{n}$ & $\%$ Fértil & \\
\hline De Sutter et al. (1996) $^{11}$ & $135 / 188$ & 71,8 & $39 / 65$ & 60,0 & 0,077 \\
Xia (1997) & $178 / 243$ & 73,3 & $147 / 218$ & 67,4 & 0,171 \\
Balaban et al. (1998) & $1589 / 2180$ & 72,9 & $620 / 864$ & 71,8 & 0,528 \\
Este artigo & $202 / 287$ & 70,4 & $413 / 611$ & 67,6 & 0,571 \\
Total & $2104 / 2898$ & 72,6 & $1219 / 1758$ & 69,3 & 0,019 \\
\hline
\end{tabular}

A total ausência de correlação entre as características do primeiro corpúsculo polar (ICP) e taxa de fertilização observada em nossos casos é consistente com os divergentes resultados segundo diversos autores (Tabela 6). Nos dados de $\mathrm{Xia}^{4}$ recalculados por nós, encontramos maior taxa de fertilização nos ovócitos com ICP íntegro $(77,2 \%)$ que nos ovócitos com ICP fragmentado $(71,1 \%)$, mas sem atingir significação estatística. Ebner et al. ${ }^{5}$ encontraram uma grande diferença a favor dos ovócitos com ICP íntegro $(87,3 \%)$ em comparação com os fragmentados (70,4\%), diferença que é significativa, ao passo que Ciotti et al. ${ }^{2}$ observaram taxa de fertilização um pouco maior em ovócitos com ICP fragmentado $(76,8 \%)$ que em aqueles com ICP íntegro $(74,8)$, mas sem atingir significação estatística. Entretanto, somando os dados dos quatro estudos, com 1218 ovócitos com ICP íntegro e 894 com ICP fragmentado, as taxas de fertilização resultam significativamente diferentes $(75,7$ e $70,6 \%$, respectivamente, $\mathrm{p}<0,005)$. Contudo, esta diferença não permite recomendar condutas clínicas diferentes com ovócitos de acordo com as características do ICP, pelas mesmas razões apresentadas quanto ao espaço perivitelino.

Tabela 6 - Taxa de fertilização segundo a presença de corpúsculo polar.

\begin{tabular}{|c|c|c|c|c|c|}
\hline \multirow[t]{3}{*}{ Autor } & \multicolumn{4}{|c|}{ Corpúsculo polar } & \multirow[t]{3}{*}{$p$} \\
\hline & \multicolumn{2}{|c|}{ Íntegro } & \multicolumn{2}{|c|}{ Fragmentado } & \\
\hline & $\mathrm{n}$ & $\%$ Fértil & $\mathrm{n}$ & $\%$ Fértil & \\
\hline Xia $(1997)^{4}$ & $217 / 281$ & 77,2 & $128 / 180$ & 71,1 & 0,140 \\
\hline Ciotti et al. (2004) ${ }^{2}$ & $333 / 445$ & 74,8 & $116 / 151$ & 76,8 & 0,624 \\
\hline Ebner et al. $(2000)^{5}$ & $315 / 361$ & 87,3 & $112 / 159$ & 70,4 & 0,025 \\
\hline Este artigo & $353 / 521$ & 67,8 & $275 / 404$ & 67,8 & 0,919 \\
\hline Total & $1218 / 1608$ & 75,7 & $631 / 894$ & 70,6 & $<0,005$ \\
\hline
\end{tabular}

A aparentemente maior taxa de fertilização dos ovócitos com citoplasma limpo comparados com ovócitos com citoplasma granulado observada em nossos casos, mas com apenas $10 \%$ de chances de ser real, foi provavelmente resultado do acaso. Apenas Serhal et al. ${ }^{7}$ encontraram alguma diferença no mesmo sentido (63,0 e 57,0\%), mas também sem significação estatística (Tabela 7). O estudo com maior número de casos, de Balaban et al. ${ }^{10}$, encontrou taxas praticamente idênticas independente da presença ou não de granulações citoplasmáticas $(72,9$ e $72,8 \%)$. Nem sequer somando todos os casos dos quatro trabalhos, com mais de 3.600 ovócitos no grupo normal e quase 750 no grupo com granulações citoplasmáticas, permite-se encontrar diferenças que sejam estatisticamente significativas: 70,4 vs $67,2 \%$, ( $\mathrm{p}=0,087)$. 
Tabela 7 - Taxa de fertilização segundo a presença de granulações citoplasmáticas.

\begin{tabular}{lccccc}
\hline Autor & \multicolumn{4}{c}{ Citoplasma } & p \\
& Normal & \multicolumn{2}{c}{ Granular } & \\
& $\mathbf{n}$ & \% Fértil & $\mathbf{n}$ & $\%$ Fértil & \\
\hline Serhal et al. (1997) & $336 / 536$ & 63,0 & $81 / 142$ & 57,0 & \\
Balaban et al. (1998) & $1589 / 2180$ & 72,9 & $115 / 158$ & 72,8 & 0,977 \\
Kahraman et al.(2000) & $91 / 134$ & 67,9 & $188 / 258$ & 72,9 & 0,304 \\
Este artigo & $529 / 765$ & 69,2 & $119 / 190$ & 62,6 & 0,10 \\
Total & $2545 / 3615$ & 70,4 & $503 / 748$ & 67,2 & 0,087 \\
\hline
\end{tabular}

O achado de menor taxa de fertilização em ovócitos com uma anomalia em comparação com os sem anomalias, mas não em relação aos com duas ou mais anomalias parece ser resultado apenas do acaso. Pelo menos outros dois autores tentaram estabelecer correlação entre o número de alterações e os resultados da fertilização $0^{5,11}$. Apesar de que houve uma tendência a menor taxa de fertilização com maior numero de alterações (até dois ou mais), as diferenças não foram significativas, como no conjunto de nossas observações (Tabela 8). Ao reunir os resultados destes autores com os nossos, portanto, com maior número de casos, houve uma diferença significativa de em torno de 10 pontos percentuais na taxa de fertilização entre os ovócitos sem alterações e os com uma e com duas ou mais anomalias morfológicas (76,9 us 65,4 e 67,7\%).

Tabela 8 - Taxa de fertilização segundo o número de alterações morfológicas do ovócito.

\begin{tabular}{lccccccccc}
\hline Autor & \multicolumn{2}{c}{ Sem anomalia } & \multicolumn{2}{c}{ 1 anomalia } & \multicolumn{2}{c}{ 2 anomalias } & \multicolumn{3}{c}{$\mathbf{p}$} \\
& $\mathbf{n}$ & \% Fértil & $\mathbf{n}$ & \% Fértil & $\mathbf{n}$ & \% Fértil & $\mathbf{0 - 1}$ & $\mathbf{0 \geq 2}$ & Conjunto \\
\hline De Sutter et al. (1996)11 & $135 / 188$ & 71,8 & $129 / 196$ & 65,8 & $78 / 124$ & 62,9 & 0,205 & 0,098 & 0,05 \\
Ebner et al. (2000) & $249 / 300$ & 83,0 & $125 / 156$ & 80,1 & $62 / 82$ & 75,6 & 0,449 & 0,117 & 0,149 \\
Este artigo & $100 / 144$ & 69,4 & $258 / 431$ & 59,9 & $252 / 373$ & 67,6 & 0,040 & 0,680 & 0,088 \\
Total & $484 / 632$ & 76,9 & $512 / 783$ & 65,4 & $392 / 579$ & 67,7 & 0,000 & 0,000 & 0,000 \\
\hline
\end{tabular}

Não encontramos outros autores que descrevessem a associação negativa entre a presença de debris e fertilização encontrada neste estudo, com diferença grande o suficiente para ser marginalmente significativa, apesar do pequeno número de casos apresentando essa alteração. De qualquer forma, justamente pela sua baixa freqüência, não seria um auxiliar importante para a seleção dos ovócitos a serem fertilizados.

Baseados em nossos resultados e nos relatados na literatura, concluímos que os parâmetros da morfologia do ovócito atualmente utilizados parecem ter alguma correlação com a taxa de fertilização, mas as diferenças entre os que apresentam alterações morfológicas e os sem essas anomalias é muito pequena para que seja de utilidade prática. A dificuldade em descartar ovócitos com alterações morfológicas torna-se ainda maior após o relato de um caso de mulher com esterilidade primária em que todos os seis ovócitos aspirados eram anormais, mas foram submetidos a ICSI, quatro fertilizaram e geraram embriões morfologicamente anormais, mas o resultado da transferência foi gravidez única com um recémnascido normal ${ }^{12}$.

Diante dessa situação surgiram novas propostas de métodos que investigam a características celulares e moleculares do ovócito. O problema com essas técnicas é que em geral são invasivas e incompativeis com manter a viabili- dade do ovócito. A técnica mais promissória descrita recentemente é a detecção do fuso meiótico (meiotic spindle detection), que pode obter-se utilizando um novo sistema de microscopia óptica computadorizada que utiliza luz polarizada, denominado polscópio (Polscope) ${ }^{13-19}$. Este método permite observar estruturas subcelulares como os microtúbulos do fuso meiótico sem afetar a viabilidade do ovócito.

No ovócito maduro o fuso deve estar alinhado com o primeiro corpúsculo polar. Desvios deste alinhamento estariam associados a menores taxas de fertilização ${ }^{19}$, porém as diferenças nas taxas de fertilização entre os com fuso meiótico "normal" e "alterado" não são muito diferentes das encontradas com as técnicas estudadas neste artigo. Portanto, ainda é cedo para saber se esta nova técnica vai permitir selecionar os ovócitos que valem a pena fertilizar dos que devem ser descartados.

Ainda não dispomos de método que permita reduzir significativamente o número de embriões excedentes nos procedimentos de fertilização assistida. O delicado equilíbrio entre oferecer ao casal a máxima probabilidade de sucesso e ao mesmo tempo evitar os embriões excedentes e a gemelaridade continua nas mãos do melhor critério de cada clínica de fertilização assistida, dentro de normas mínimas de segurança. É provável que, com o progresso das pesquisas e melhores méto- 
dos, seja possível no futuro separar os ovócitos com possibilidades de fertilização quase perfeitas, mas por enquanto temos que aceitar a necessidade de fertilizar pelo menos três ovócitos para conseguir um ou dois embriões que possam ser transferidos.

\section{Referências}

1. Ebner T, Moser M, Yaman C, Feichtinger O, Hartl J, Tews G. Elective transfer of embryos selected on the basis of first polar body morphology is associated with increased rates of implantation and pregnancy. Fertil Steril. 1999;72(4):599-603.

2. Ciotti PM, Notarangelo L, Morselli-Labate AM, Felletti V, Porcu E, Venturoli S. First polar body morphology before ICSI is not related to embryo quality or pregnancy rate. Hum Reprod. 2004;19(10):2334-9.

3. Loutradis D, Drakakis P, Kallianidis K, Milingos S, Dendrinos S, Michalas S. Oocyte morphology correlates with embryo quality and pregnancy rate after intracytoplasmic sperm injection. Fertil Steril. 1999;72(2):240-4

4. Xia P. Intracytoplasmic sperm injection: correlation of oocyte grade based on polar body, perivitelline space and cytoplasmic inclusions with fertilization rate and embryo quality. Hum Reprod. 1997;12(8):1750-5.

5. Ebner T, Yaman C, Moser M, Sommergruber M, Feichtinger O, Tews G. Prognostic value of first polar body morphology on fertilization rate and embryo quality in intracytoplasmic sperm injection. Hum Reprod. 2000;15(2):427-30.

6. Kahraman S, Yakin K, Dönmez E, Samli H, Bahce M, Cengiz G, et al. Relationship between granular cytoplasm of oocytes and pregnancy outcome following intracytoplasmic sperm injection. Hum Reprod. 2000;15(11):2390-3.

7. Serhal PF, Ranieri DM, Kinis A, Marchant S, Davies M, Khadum IM. Oocyte morphology predicts outcome of intracytoplasmic sperm injection. Hum Reprod. 1997;12(6):1267-70.

8. Alikani M, Palermo G, Adler A, Bertoli M, Blake M, Cohen J. Intracytoplasmic sperm injection in dysmorphic human oocytes. Zygote. 1995;3(4):2838.
9. Sousa M, Tesarik J. Ultrastructural analysis of fertilization failure after intracytoplasmic sperm injection. Hum Reprod. 1994;9(12):2374-80.

10. Balaban B, Urman B, Sertac A, Alatas C, Aksoy S, Mercan R. Oocyte morphology does not affect fertilization rate, embryo quality and implantation rate after intracytoplasmic sperm injection. Hum Reprod. 1998;13(12):3431-3.

11. De Sutter P, Dozortsev D, Qian C, Dhont M. Oocyte morphology does not correlate with fertilization rate and embryo quality after intracytoplasmic sperm injection. Hum Reprod. 1996;11(3):595-7.

12.Esfandiari N, Ryan EA, Gotlieb L, Casper RF. Successful pregnancy following transfer of embryos from oocytes with abnormal zona pellucida and cytoplasm morphology. Reprod Biomed Online. 2005; $11(5): 620-3$.

13.Wang WH, Meng L, Hackett RJ, Keefe DL. Developmental ability of human oocytes with or without birefringent spindles imaged by Polscope before insemination. Hum Reprod. 2001;16(7):14648.

14. Moon JH, Hyun CS, Lee SW, Son WY, Yoon SH, Lim JH. Visualization of the metaphase II meiotic spindle in living human oocytes using the Polscope enables the prediction of embryonic developmental competence after ICSI. Hum Reprod. 2003;18(4):81720.

15. Rienzi L, Ubaldi F, Martinez F, Iacobelli M, Minasi MG, Ferrero S, et al. Relationship between meiotic spindle location with regard to the polar body position and oocyte developmental potential after ICSI. Hum Reprod. 2003;18(6):1289-93.

16. Cohen Y, Malcov M, Schwartz T, Mey-Raz N, Carmon A, Cohen T, et al. Spindle imaging: a new marker for optimal timing of ICSI? Hum Reprod. 2004;19(3):649-54.

17. Coticchio G, Sereni E, Serrão L, Mazzone S, Iadarola I, Borini A. What criteria for the definition of oocyte quality? Ann N Y Acad Sci. 2004;1034:132-44.

18. De Santis L, Cino I, Rabellotti E, Calzi F, Persico P, Borini A, et al. Polar body morphology and spindle imaging as predictors of oocyte quality. Reprod Biomed Online. 2005;11(1):36-42.

19. Rienzi L, Ubaldi F, Iacobelli M, Minasi MG, Romano $\mathrm{S}$, Greco E. Meiotic spindle visualization in living human oocytes. Reprod Biomed Online. 2005;10(2):192-8. 